\title{
A Library of Generic Algorithms in Ada
}

\author{
David R. Musser \\ General Electric Company \\ Corporate Research \& Development \\ P. O. Box 8 \\ Schenectady, New York 12301
}

\author{
Alexander A. Stepanov \\ Polytechnic University \\ Computer Science Department \\ 333 Jay Street \\ Brooklyn, New York 11201
}

\begin{abstract}
It is well-known that data abstractions are crucial to good software engineering practice. We argue that algorithmic abstractions, or generic algorithms, are perhaps even more important for software reusability. Generic algorithms are parameterized procedural schemata that are completely independent of the underlying data representation and are derived from concrete, efficient algorithms. We discuss this notion with illustrations from the structure of an Ada library of reusable software components we are presently developing.
\end{abstract}

\section{Introduction}

\subsection{Purpose of the library}

The purpose of the Ada Generic Library is to provide an Ada programmer with powerful generic packages for data structures such as lists, matrices, strings, trees, and graphs, along with numerical and combinatorial algorithms. Our main goal in this introduction is to explain both the structure of this particular library and the general principles we have followed in creating that structure. We believe these principles, which are quite different from those on which other libraries such as in [1] have been founded, have broad applicability to the goal of widely-usable software components in Ada.

The first phase of the library concentrates on a significant subset of the data structures problem: an extensive set of linear data structure manipulation facilities for different kinds of linked lists and vectors

Permission to copy without fee all or part of this material is granted provided that the copies are not made or distributed for direct commercial advantage, the ACM copyright notice and the title of the publication and its date appear, and notice is given that copying is by permission of the Association for Computing Machinery. To copy otherwise, or to republish, requires a fee and / or specific permission.

(C) 1987 ACM 0-89791-243-8/87/0012/0216

$\$ 1.50$ (one dimensional arrays). The data structures and algorithms included have been selected based on their well-established usefulness in a wide variety of of applications. Over 300 subprograms will be provided in the first phase of the library, in eleven Ada packages. (In the current release, eight packages containing over 150 subprograms are included.) This development is a part of the Reusable Ada Repository System, a joint project of GE's Western Systems (Sunnyvale, California) and Corporate Research and Development.

\subsection{Principles behind the library}

The main principles we have followed in building the library are the following:

1. Extensive use of generic algorithms, such as generic sort and merge algorithms that can be specialized to work for many different data representations and comparison functions.

2. Building up functionality in layers, separating, to as large an extent as possible, concerns about representations from those of algorithms.

3. Obtaining high efficiency in spite of the layering (using Ada's inline compiler directive).

4. Emphasis on careful selection and programming of highly efficient algorithms.

5. High quality documentation that makes it easy to find operations in the library and select the best algorithm and data structure for the application at hand.

The most important technical idea is that of generic algorithms, which are a means of providing functionality in a way that abstracts away from details of representation and basic operations. Instead of referring directly to the host language facilities, generic algorithms are defined in terms a few primitive operations that are considered to be parameters. By plugging in actual 
operations for these parameters, one obtains specific instances of the algorithms for a specific data structure. By carefully choosing the parameterization and the algorithms, one obtains in a small amount of code the capability to produce many different useful operations. It becomes much easier to obtain the operations needed for a particular application by plugging components together than it would be to program them directly.

\subsection{Related technology}

The notion of generic algorithms is not entirely new, but there has not been any attempt to structure a general software library founded on this idea. Older program libraries, written in Fortran or other languages without the facilities for generic programming, could not take advantage of the algorithm abstractions that were known. But even the recent improvements in abstraction facilities in contemporary programming languages, such as Ada, have not precipitated widespread use of algorithmic abstraction. (Booch, for example, makes some use of generic algorithms for list and tree structures, but almost as an afterthought in a chapter on utilities.) For the benefits of this approach to be fully realized, great care must be exercised in selecting and structuring algorithms, especially in determining how they are parameterized and how they are used to develop more concrete levels of the library. Indeed, we view algorithm selection, abstraction, and structuring as being of far greater importance to software reusability than any language or other human-interface issues; experience with Unix tools provides ample evidence of this point.

\section{Structure of the Library}

The key structuring mechanism used in building the library is abstraction. We discuss four classes of abstractions that we have found useful in structuring the library, as shown in Table 1, which lists a few examples of packages in the library. Each of these Ada packages has been written to provide generic algorithms and generic data structures that fall into the corresponding abstraction class. (The packages marked with a $*$ are not included in the current release of the library.) Brief definitions of the abstraction classes are given in the table and are illustrated in Figure 1.

\subsection{Data abstractions}

Data abstractions are data types and sets of operations defined on them (the usual definition); they are abstractions mainly in that they can be understood (and formally specified by such techniques as algebraic axioms) independently of their actual implementation. In Ada, data abstractions can be written as packages which define a new type and procedures and functions on that type. Another degree of abstractness is achieved by using a generic package in which the type of elements being stored is a generic formal parameter. In our library, we program only a few such data abstractions directly - those necessary to create some fundamental data representations and define how they are implemented in terms of Ada types such as arrays, records and access types. Three such packages, which we refer to as "low-level data abstraction packages," are included in the current library. Most other data abstractions are obtained by combining existing data abstraction packages with packages from the structural or representational classes defined below.

\subsection{Algorithmic abstractions}

These are families of data abstractions that have a set of efficient algorithms in common; we refer to the algorithms themselves as generic algorithms. For example, in our library there is a package of generic algorithms for linked-lists; in a future release there will be a more general package of sequence algorithms whose members can be used on either linked-list or vector representations of sequences. The linked-list generic algorithms package contains 31 different algorithms such as, for example, generic merge and sort algorithms that are instantiated in various ways to produce merge and sort subprograms in structural abstraction packages such as singly-linked lists and doubly-linked lists.

We stress that the algorithms at this level are derived by abstraction from concrete, efficient algorithms. As an example of algorithmic abstraction, consider the task of choosing and implementing a sorting algorithm for linked list data structures. The merge sort algorithm can be used and, if properly implemented, provides one of the most efficient sorting algorithms for linked lists. Ordinarily one might program this algorithm directly in terms of whatever pointer and record field access operations are provided in the programming language. Instead, however, one can abstract away a concrete representation and express the algorithm in terms of the smallest possible number of generic operations. In this case, we essentially need just three operations: Next and Set_Next for accessing the next cell in a list, and Is_End for detecting the end of a list. For a particular representation of linked lists, one then obtains the corresponding version of a merge sorting algorithm by instantiating the generic access operations to be subprograms that access that representation. 


\begin{tabular}{|c|c|}
\hline $\begin{array}{l}\text { Data Abstractions } \\
\text { Data types with operations } \\
\text { defined on them }\end{array}$ & $\begin{array}{c}\text { System_Allocated_Singly_Linked } \\
\text { User_Allocated_Singly_Linked } \\
\text { \{Instantiations of representational abstractions\} }\end{array}$ \\
\hline $\begin{array}{l}\text { Algorithmic Abstractions } \\
\text { Families of data abstractions } \\
\text { with common algorithms }\end{array}$ & $\begin{array}{c}\text { Sequence_Algorithms* } \\
\text { Linked_List_Algorithms } \\
\text { Vector_Algorithms }\end{array}$ \\
\hline $\begin{array}{l}\text { Structural Abstractions } \\
\text { Intersections of } \\
\text { algorithmic abstractions }\end{array}$ & $\begin{array}{c}\text { Singly_Linked_Lists } \\
\text { Doubly_Linked_Lists* } \\
\text { Vectors* }\end{array}$ \\
\hline $\begin{array}{l}\text { Representational Abstractions } \\
\text { Mappings from one structural } \\
\text { abstraction to another }\end{array}$ & $\begin{array}{c}\text { Double_Ended_Lists } \\
\text { Stacks } \\
\text { Output_Restricted_Deques }\end{array}$ \\
\hline
\end{tabular}

Table 1: Classification of Abstractions and Example Ada Packages

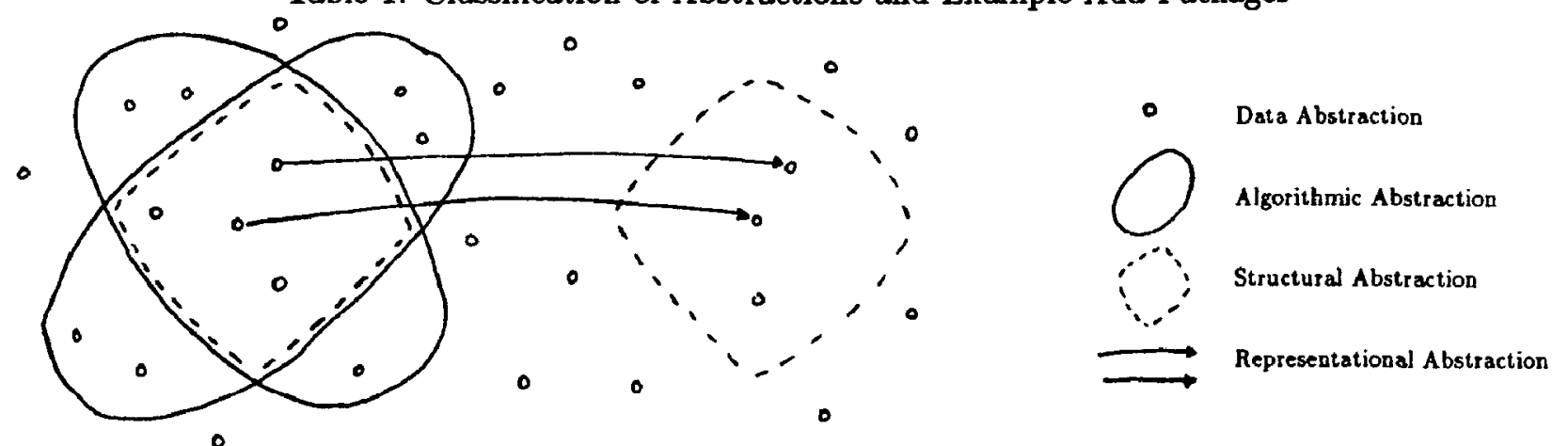

Figure 1: Kinds of Abstractions Used in Structuring the Library

Thus in Ada one programs generic algorithms in a generic package whose parameters are a small number of types and access operations-e. g.,

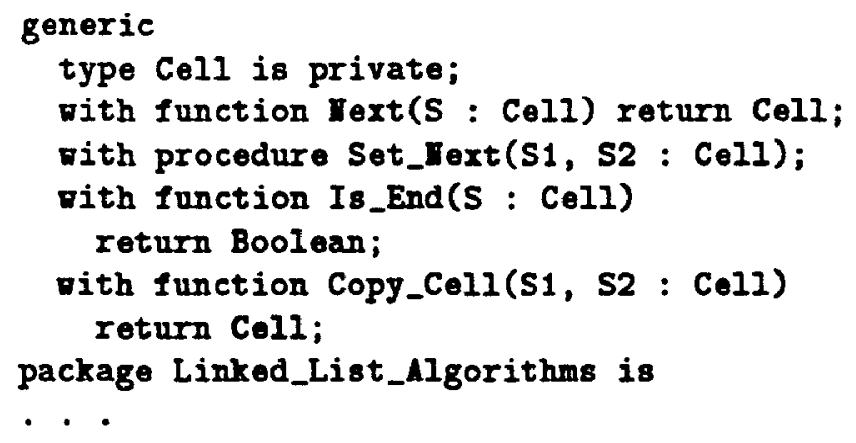

The subprograms in the package are algorithms such as Merge and Sort that are efficient when Hext, Set_lext, etc., are instantiated with constant time operations.

\subsection{Structural abstractions}

Structural abstractions (with respect to a given set of algorithmic abstractions) are also families of data abstractions: a data abstraction $A$ belongs to a structural abstraction $S$ if and only if $S$ is an intersection of some of the algorithmic abstractions to which $A$ belongs.
An example is singly-linked-lists, the intersection of sequence- , linked-list-, and singlylinkedlistalgorithmic abstractions. It is a family of all data abstractions that implement a singly-linked representation of sequences (it is this connection with more detailed structure of representations that inspires the name "structural abstraction"). (In the current release, the Singly. Linked Lists package is actually programmed just in terms of the Linked_List_Algorithms package.)

Note that, as an intersection of algorithmic abstractions, such a family of data abstractions is smaller than the algorithm abstraction classes in which it is contained, but a larger number of algorithms are possible, because the structure on which they operate is more completely defined.

Programming of structural abstractions can be accomplished in Ada with the same kind of generic package structure as for generic algorithms. The SinglyLinked Lists package contains 66 subprograms, most of which are obtained by instantiating or calling in various ways some member of the Linked_ist. 11gorithms package. In Ada, to actually place one data abstraction in the singly-linked-lists family, one instantiates the Singly Linked_Lists package, using as actual parameters a type and the set of operations 
on this type from a data abstraction package such as System_Allocated_Singly_Linked that defines an appropriate representation.

\subsection{Representational abstractions}

These are mappings from one structural abstraction to another, creating a new type and implementing a set of operations on that type by means of the operations of the domain structural abstraction. For example, stacks can easily be obtained as a structural abstraction from a sequence structural abstraction, and this is carried out in Ada using generic packages in a manner that will be demonstrated in the Appendix. Note that what one obtains is really a family of stack data abstractions, whereas the usual programming techniques give only a single data abstraction.

\section{Linear Data Structures}

\subsection{Low-level data abstractions}

In the current release of the library we have provided three different low-level data abstractions using singlylinked list representations:

- The System_Allocated_Singly_Linked package provides records containing datum and link fields, allocated using the standard heap allocation and deallocation procedures.

- Once_User_Allocated_Singly_Linked provides more efficient allocation and deallocation by allocating an array of records as a storage pool, but is less flexible than the system allocated package since this array and the system heap are managed separately.

- Auto_Reallocating_Singly_Linked also uses an array of records for efficiency but automatically allocates a larger array whenever necessary; its disadvantage is that the parameters controlling the reallocation may need to be tuned to achieve optimum reallocation behavior.

\subsection{Algorithmic, structural and repre- sentational abstractions}

The current release of the library provides the following algorithmic, structural and representational abstraction packages:

- SinglyLinked_Lists is a structural abstraction package that provides over 60 subprograms for operations on a singly-linked list representation, including numerous kinds of concatenation, deletion, substitution, searching and sorting operations (the selection is based mainly on Common Lisp facilities [7]).

- Linked_List_Algorithms is a generic algorithms package that is the source of most of the algo rithms used in Singly_inked_Lists; many of the same algorithms will be. used in implementing the Doubly Linked_Lists package.

- Stacks provides the familiar linear data structure in which insertions and deletions are restricted to one end.

- Double_Ended_Lists employs header cells with singly-linked lists to make some operations such as concatenation more efficient and to provide more security in various computations with lists.

\section{- Output_Restricted_Deques}

provides a data structure that restricts insertions to both ends and deletions to one end, making use of Double_Ended Lists.

The latter three packages are representational abstractions that produce different structural abstractions from different representations of sequences. In particular, any of the three different low-level representations of singly-linked-lists provided can easily be plugged together with any of these three representational abstractions, as well as with the Singly_Linked_Lists package, for a total of 12 different possible combinations. Each of these 12 combinations, called a Partially Instantiated Package, or PIP for short, is included in the library. To use one of them, one only has to instantiate the element type, and perhaps some configuration parameters, to specific values.

A later release will also include:

- Sequences

- Doubly Linked_ists

- Simple_vectors

- Extensible_Vectors

packages, along with several low-level data abstraction packages that plug together with them.

\section{Selection from the library}

The first observation we would make is that proper classification of software components for maximum usability may well depend more on internal structure than on functional (input-output) behavior. In searching the library, the programmer needs to know not only 
whether there is a subprogram that performs the right operation, but also what kind of data representation it uses (if it is not a completely generic algorithm), since in all but the simplest cases it will be used in a particular context that may strongly favor one representation over another.

Experienced programmers will sometimes want to use generic algorithms directly, instantiating the generic access operations to be subprograms accessing a particular data representation. Although generic, these algorithms are tailored to be used with data representations with particular complexity characteristics, such as linked-list- versus array-like representations, and the programmer must be aware of these issues.

This is not to say that intelligent use of the library necessarily requires the programmer to examine the bodies of the subprograms. If construction of the library is, as we have recommended, algorithmicallydriven and draws upon the best books and articles on algorithms and data structures, then it should be possible to develop sufficiently precise and complete selection criteria based on the advice in those books and articles. Again, the preparation of these selection criteria and other documentation must be done very carefully and thoroughly to make later usage by programmers as simple as possible.

With the current Linear Data Structures library, there are, at a minimum, three kinds of selections to be made:

1. the choice of a low-level data abstraction package

2 . the choice of a structural or representational abstraction package

3. the choice of operations within the structural or representational package

The fact that the structure of our library allows separate choices for 1 and 2 means that there are many more selections available than would be the case with more conventional organizations. However, it is not the case that these choices are entirely independent of each other or of the choices in 3. In fact, the programmer will often have to give careful consideration to the the combination of operations that he or she expects to use in an application, and make a package selection based on algorithmic issues of time and space efficiency of the subprograms as documented in the subprogram descriptions. Another issue that might dictate a choice would be the possible exceptions raised by the operations to be used.

\section{Conclusions and future work}

In summary, the main points we want to make are:
- that to achieve truly reusable software components, extensive use should be made of algorithmic abstraction;

- that, indeed, development of a software library should be algorithmically driven;

- and that careful development of algorithms, data structures, and selection criteria are essential to the success of the library.

The library we are developing in Ada is a significant attempt to implement this approach. We have, with Aaron Kershenbaum of Polytechnic University, also experimented extensively with the generic algorithms approach in Scheme, using higher order procedures; and we have implemented a number of useful generic subprograms in $\mathrm{C}$.

We have only recently begun actual construction of the library discussed in this paper; thus it is difficult to predict the full scope of this work. A library of the nature we have discussed could be very large-eventually containing hundreds of packages, each containing from a few to perhaps a hundred subprograms. How, it may be asked, will it be possible to make effective use of such a large library?

We believe there is no easy answer to this question, but the organization by abstraction classes discussed herein should substantially reduce the size of the library and aid in making effective use of it. As programmers gain experience with use of even a few of the packages they can begin learning the general structure the library, which will greatly assist in intelligent selection from and proper use a wide range of library components. Among all of the technologies being explored today to improve software productivity, it may well be that a well-structured library of generic components offers the greatest benefits.

\section{References}

[1] Booch, G., Software Components in Ada. Benjamin/Cummings, 1987.

[2] O.-J. Dahl, E. W. Dijkstra, and C. A. R. Hoare, Structured Programming, Academic Press, 1972.

[3] Brian W. Kernighan and P. J. Plauger, "Software Tools in Pascal," Addison-Wesley, 1981.

[4] Donald E. Knuth, The Art of Computer Programming, Vols. 1-3, Addison-Wesley, 1968, 1969, 1973.

[5] D. R. Musser and A. A. Stepanov, Ada Generic Library Linear Data Structure Packages, Vol. 1, to appear as a General Electric Corporate Research and Development Report, October 1987. 
[6] R. Sedgewick, Algorithms, Addison-Wesley, 1983.

[7] Guy L. Steele, Common LISP: The Language, Digital Press, 1984.

[8] Niklaus Wirth, Algorithms + Data Structures = Programs, Prentice-Hall, 1976.

\section{A Appendix: Examples of li- brary structure}

At the algorithmic abstraction level, our library includes a package called Linked_List_algorithms containing numerous algorithms for basic operations on linked lists that are efficient provided that the representation satisfies certain requirements. It can be described as a collection of many useful algorithms expressible in terms of Iext, Set_lext, Is_End, and Copy_Cell operations that are assumed to take constant time. Using Linked_List_1lgorithms, other packages provide structural abstractions that assume more details about particular representations: SingIy_LinkedLIsts and Doubly_LinkedLists. We will examine a small number of the subprograms at these levels and see how they are used to build more concrete packages.

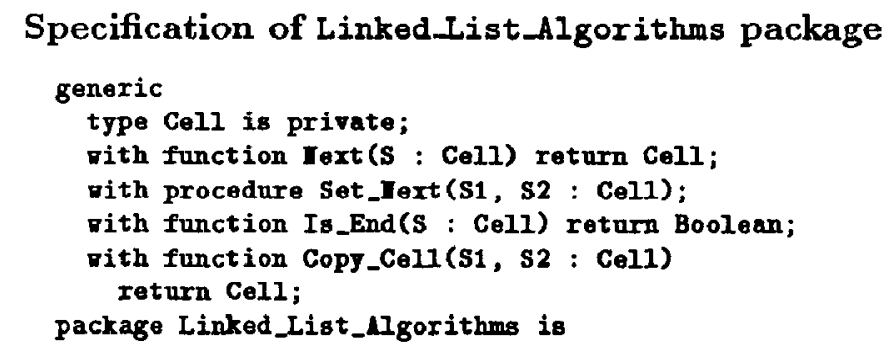

function Reverse_Lppend(s1, s2 : Cell) return Cell;

-- Returns a sequence consisting of the

-- lements of $\mathrm{S1}$, in reverse order,

-- folloged by those of $\mathrm{S} 2$ in order.

generic

Dith function Test (X : Cell)

return Boolean;

function Count (s : Cell) return Integer;

-- Returns a non-negative integer, the

-- number of elements $E$ of $S$ such that

-- Test(E) is true.

generic

vith function Test( $X, Y$ : Cell) return Boolean;

vith procedure Free(X : CELL);

function Delete_Duplicates(s : Cell)

return Ce11;

- Returns a sequence of the elements

-- of $S$ but vith only one occurrence of

-- each, using Test $(X, Y)$ as the test for

- equality.

. .

end Linked_List_Algorithms;

We have shown only three of the 31 subprograms in this package.

\section{Body of Linked_List_Algorithms package}

In the package body an auxiliary function called Advance is introduced and used in many of the algorithms.

package body Linked_List_11gorithes is

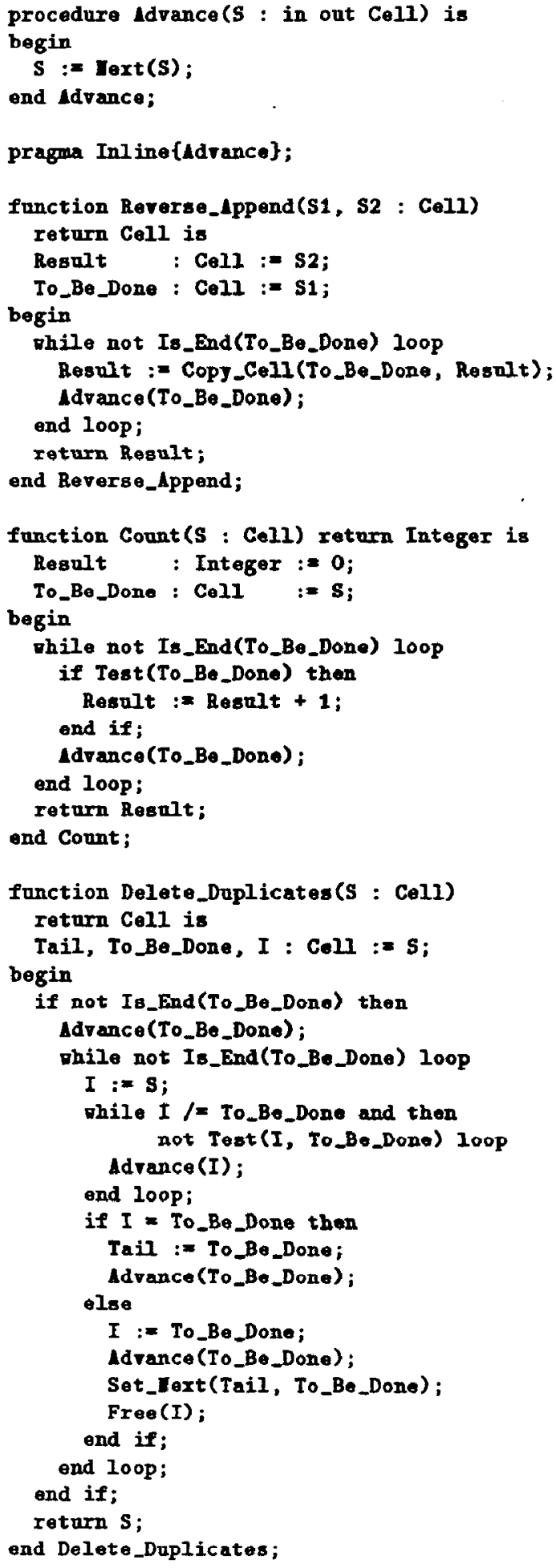

ond Linked_List_Algorithms; 


\section{Specification of Singly_Linked_Lists}

This structural abstraction is expressed in terms of primitive operations for list access that will be provided by a low-level data abstraction package (they are named with the character 0 appended so that it is possible to use renamings to export these operations.)

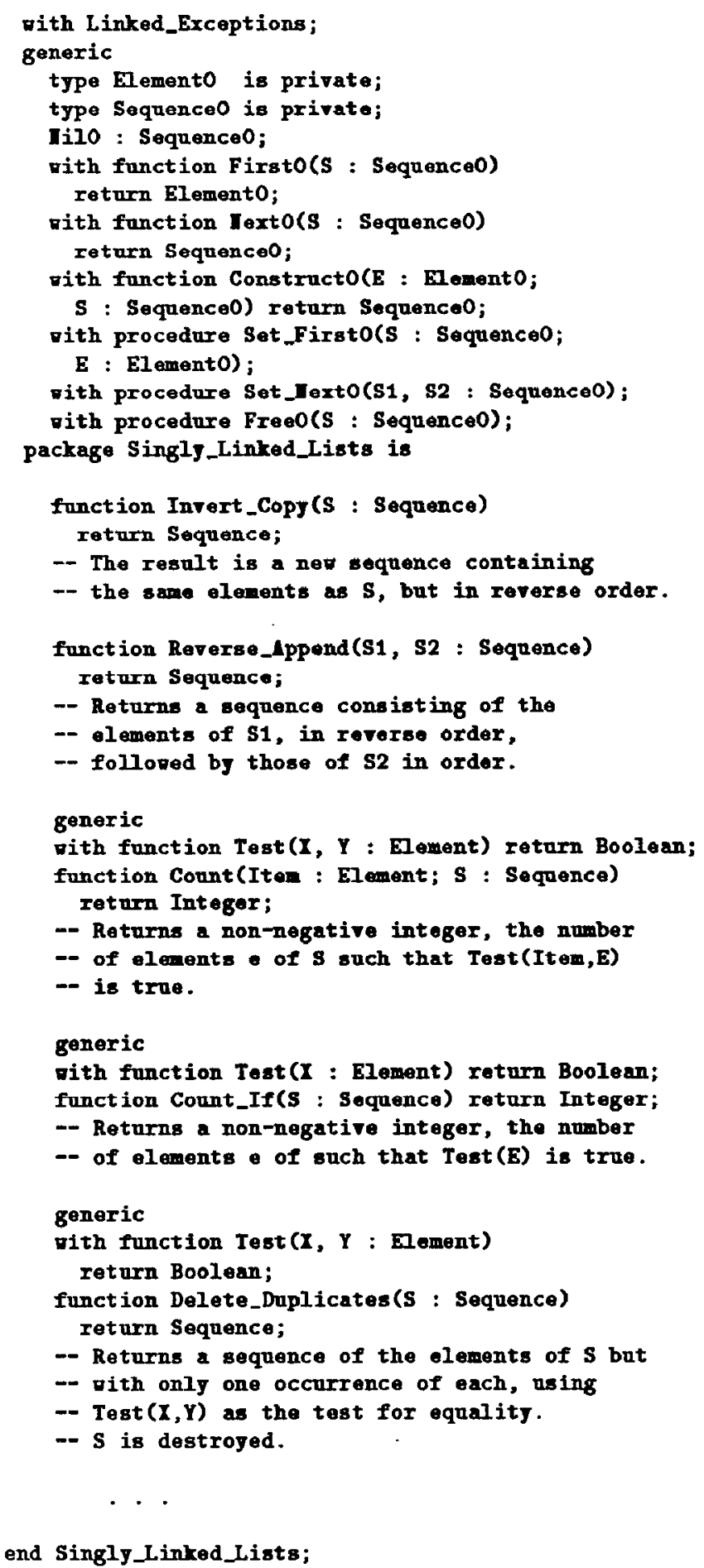

Here we have listed only a few of the 66 subprograms in the package.

\section{Body of Singly_inked_Lists}

The package body illustrates how many of the subprograms can be built with different instantiations of basic algorithms from Linked_List_Algorithms:

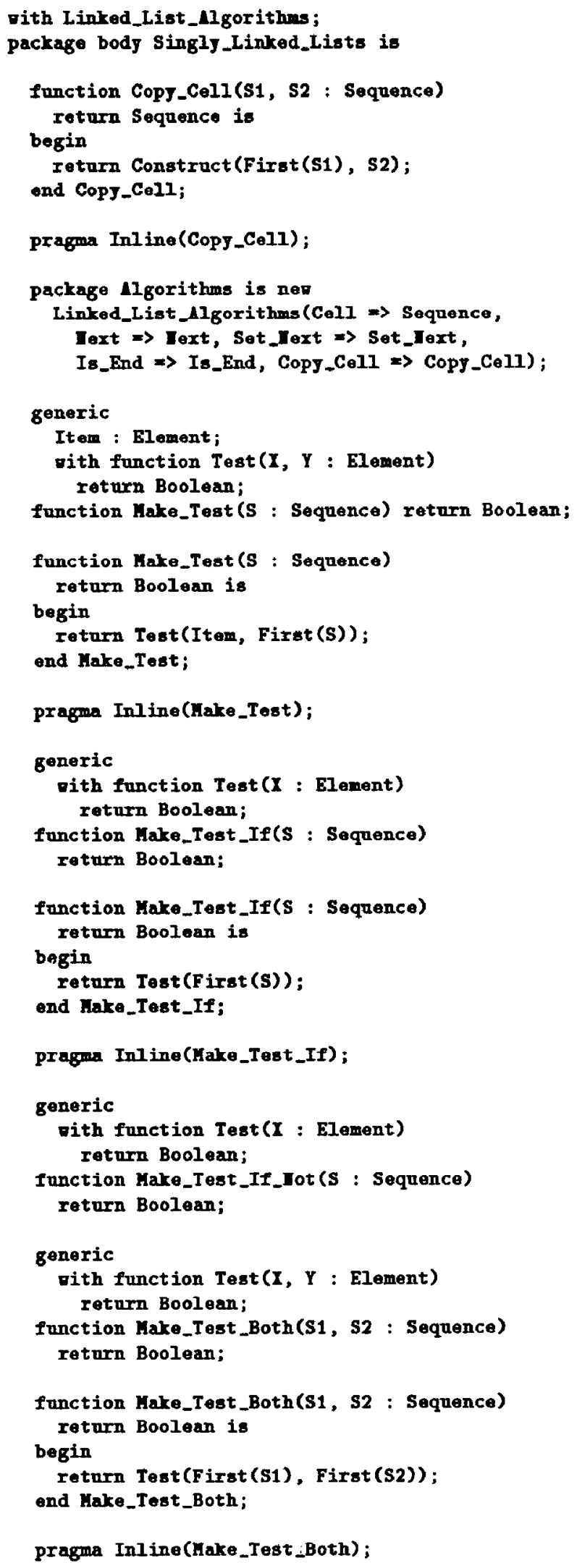




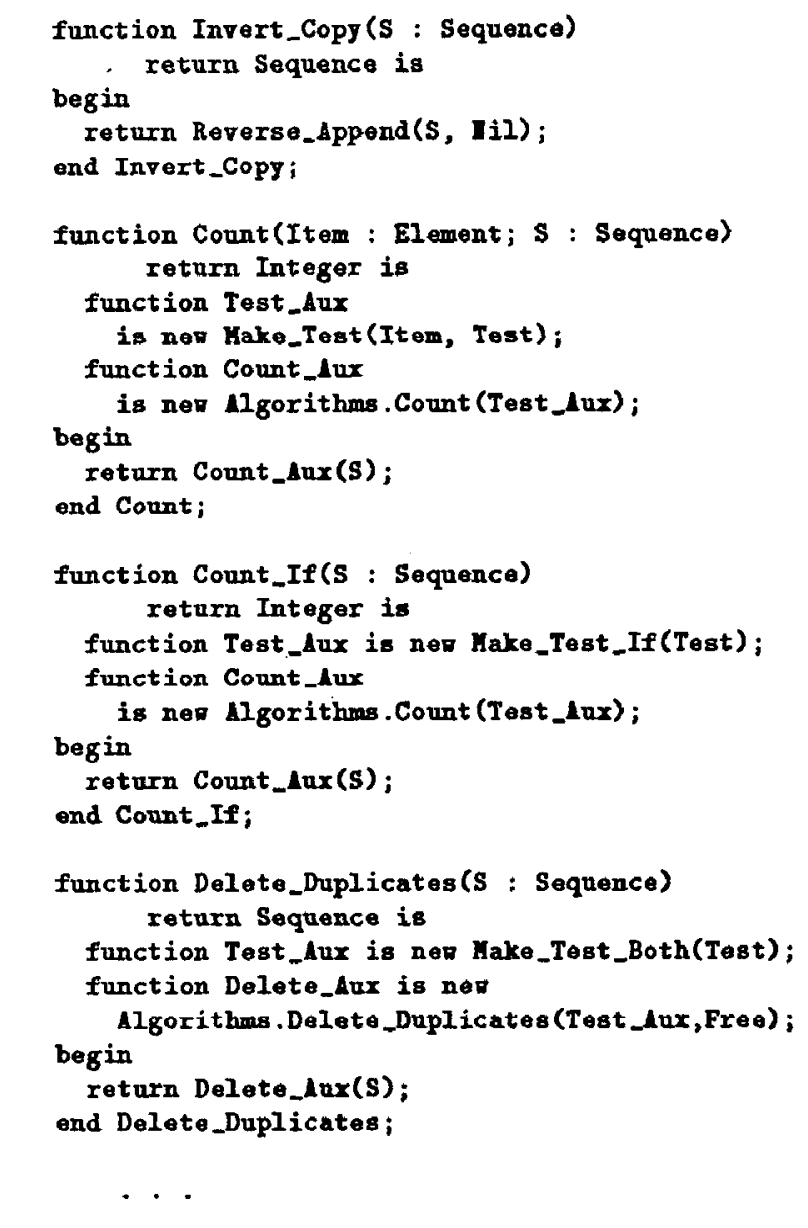

end Singly_Linked_Lists;

\section{Specification of System_Allocated_Singly_Iinked}

Now we introduce an actual data representation for singly-linked lists, by first creating a package defining just a simple record structure and corresponding subprograms for creating and accessing this structure. In this package the standard heap allocar tion and deallocation procedures are used, but our library also includes two other packages defining data representations that provide a more elaborate allocation method for the same representation.

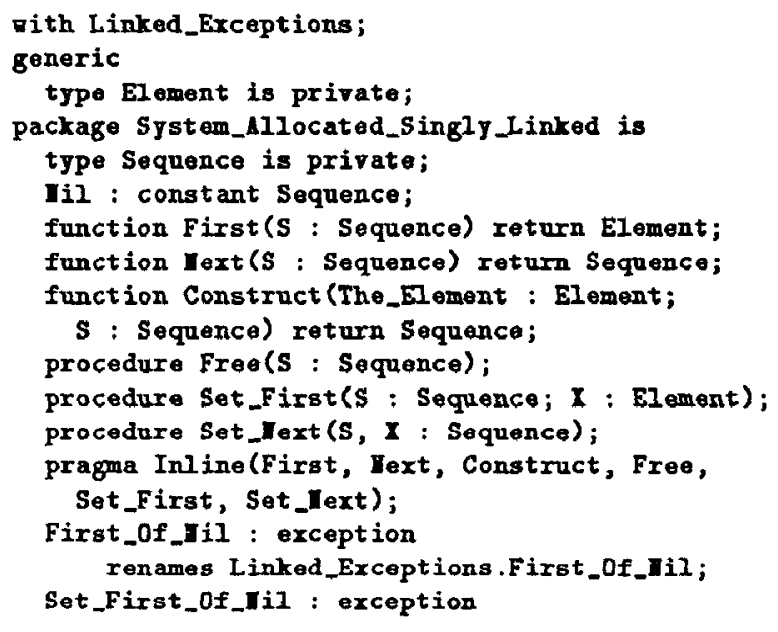

renames Linked_Except ions.Set_First_0f_Iil;

Iext_Of_Iil : exception

renames Linked_Exceptions . Dext_Of_lil;

Set_Iext_Of_Iil : exception

renames Linked_Exceptions. Set_lext_Of_Iil;

Out_Of_Construct_Storage : exception

renames

Linked_Exceptions.0ut_Of_Construct_Storage;

private

type Iode;

type Sequance is access Inade;

Iil : constant Sequence : = null;

ond System_lllocated_Singly_linked;

\section{Body of System_Allocated_Singly_Linked}

vith Unchecked_Derllocation;

package body Systan_lllocated_Singly_Linked is

type Iode is record

Datum : Element;

Link : Sequence;

end record;

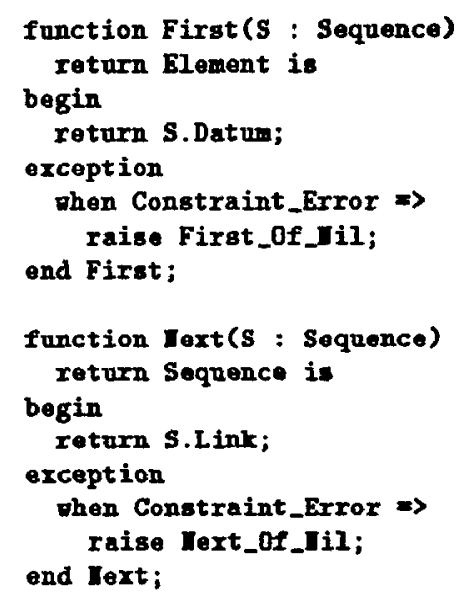

function Construct (The_Blement : Element;

S : Sequence) return Sequence is

begin

return nev Jode'(The_Elesent, S);

exception

then Storage_Error $\Rightarrow$

raise Out_of_Construct_Storage;

and Construct;

procedare Free_dux is ned

Unchecked_Deallocation(Iode, Sequence);

procedure Free(s : Sequence) is

Temp : Sequence : $=s$;

begin

Free_Aux (Temp);

and Free;

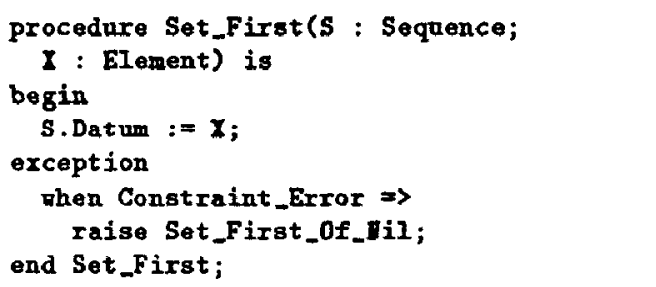


procedure Set_llext(s, $x$ : Seguence) 1:

begin

$$
\text { S.Link := } \mathbf{X} \text {; }
$$

exception

when Constraint_Error $\Rightarrow$

raise Set_llext_of_Iil;

end Set_llext;

end System_Allocated_Singly_Iinked;

\section{Partially Instantiated Package (PIP)}

Next comes the step of plugging these packages together, in a Partially Instantiated Package that has only the Element type as a generic parameter:

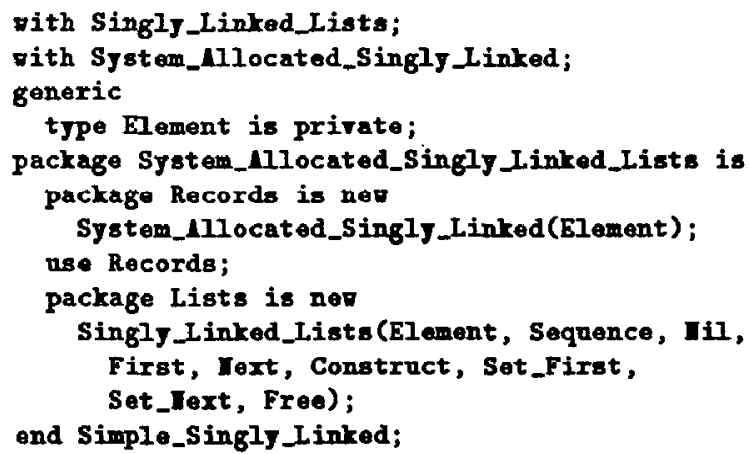

\section{Instantiation with type Integer}

Note that we have a package that is still generic in the type of elements stored as data in the lists. To illustrate how to make use of the list processing capabilities that have been built up, we next carry out an instantiation of the element type as Integer.

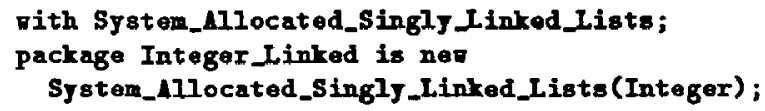

\section{Test suite for Singly Linked Lists}

We show a small part of an extensive test suite we have developed for Singly_Linked_ists, using the Integer_Linked instance.

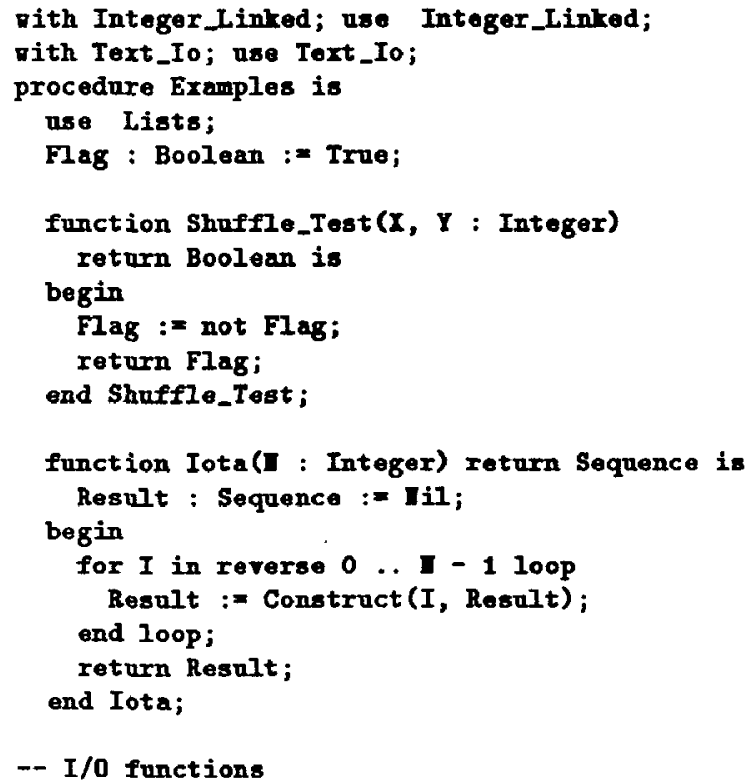

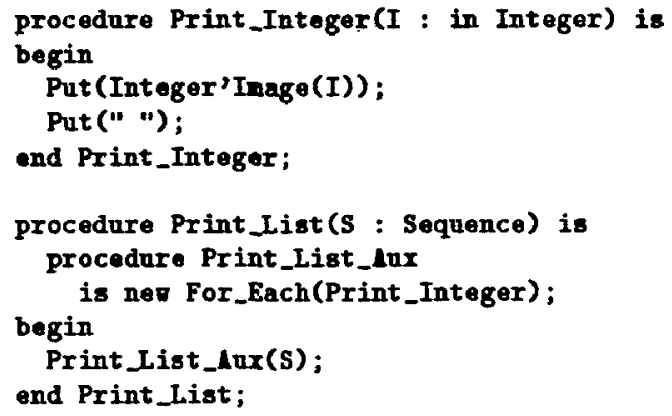


end;

end Examples;

\section{Output from the tests}

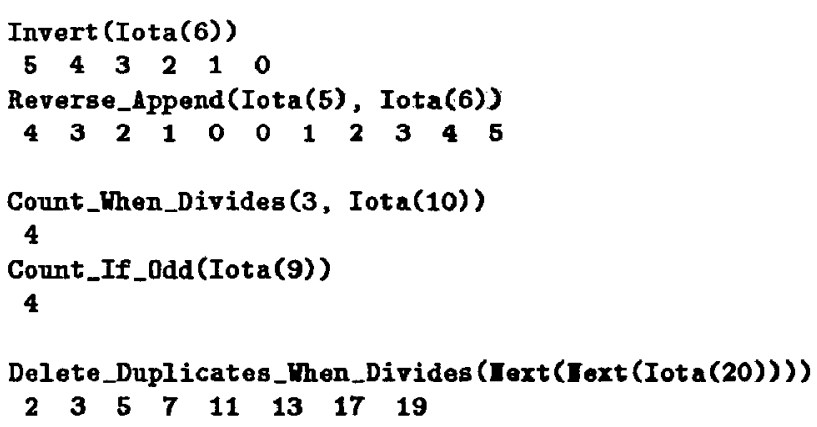

Though limited to a thin vertical slice of the library structure, these examples show many of the features of our approach and the potential for a programmer to make use of different abstraction mechanisms; e.g., to use the Singly_Linked_List package in conjunction with his or her own more complex record structure to produce a large collection of useful algorithms operating on that structure, just by plugging them together as we have done to produce the System_lllocated_Singly_Linked_Lists package.

\section{Representational abstraction example}

In order to illustrate representational abstractions, we give the following treatment of stacks:

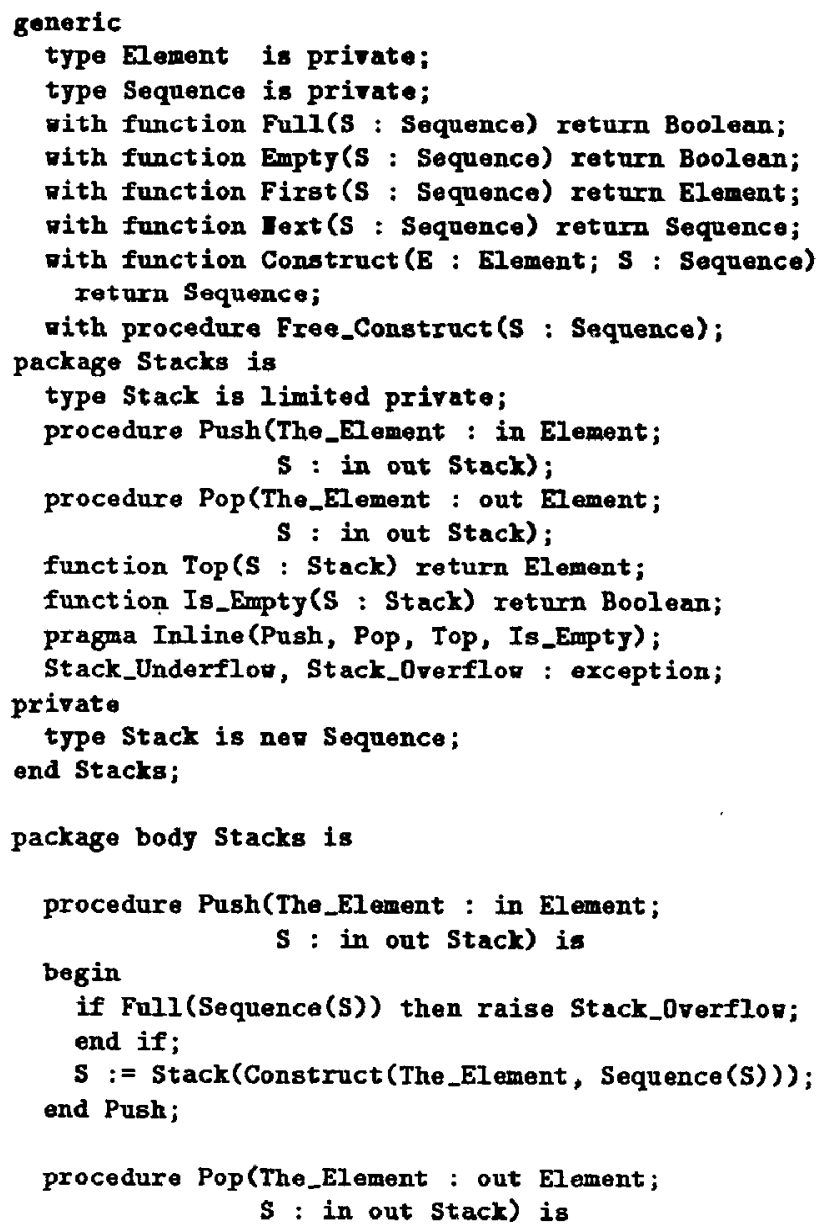

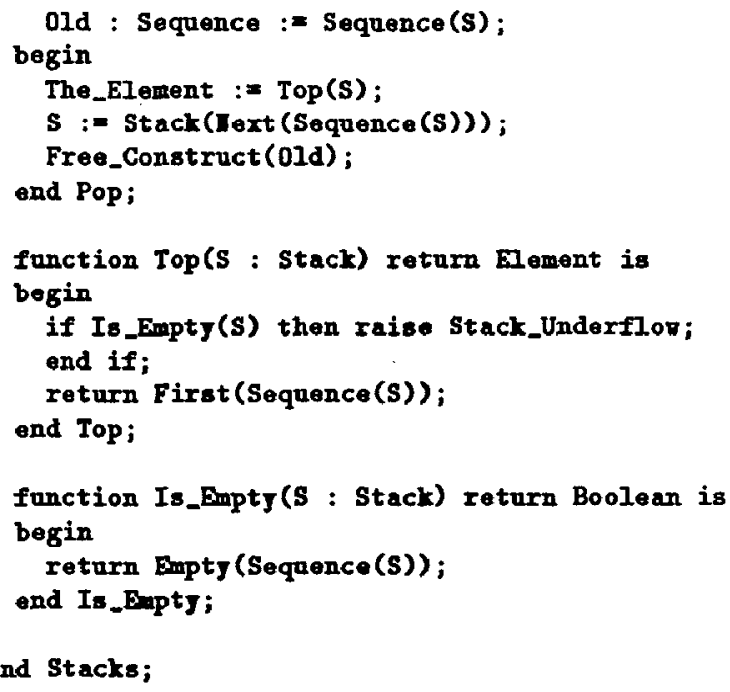

Here we have created a stack structural abstraction by a simple mapping that allows the operations of a sequence structural abstraction to be used to implement those of stacks. Again, we emphasize that this approach yields a whole family of stack data abstractions, one for each possible sequence data abstraction, including all vector as well as linked list representations. 